\title{
A cyber-anima-based model of material conscious information network
}

Jianping Shen and Yadong Huang

\author{
Yueting Chai \\ National Engineering Laboratory for E-commerce Technology, \\ Tsinghua University, Beijing, China
}

\begin{abstract}
Purpose - This paper aims to study the node modeling, multi-agent architecture and addressing method for the material conscious information network (MCIN), which is a large-scaled, open-styled, self-organized and ecological intelligent network of supply-demand relationships.
\end{abstract}

Design/methodology/approach - This study models the MCIN by node model definition, multi-agent architecture design and addressing method presentation.

Findings - The prototype of novel E-commerce platform based on the MCIN shows the effectiveness and soundness of the MCIN modeling. By comparing to current internet, the authors also find that the MCIN has the advantages of socialization, information integration, collective intelligence, traceability, high robustness, unification of producing and consuming, high scalability and decentralization.

Research limitations/implications - Leveraging the dimensions of structure, character, knowledge and experience, a modeling approach of the basic information can fit all kinds of the MCIN nodes. With the double chain structure for both basic and supply-demand information, the MCIN nodes can be modeled comprehensively. The anima-desire-intention-based multi-agent architecture makes the federated agents of the MCIN nodes self-organized and intelligent. The MCIN nodes can be efficiently addressed by the supplydemand-oriented method. However, the implementation of the MCIN is still in process.

Practical implications - This paper lays the theoretical foundation for the future networked system of supply-demand relationship and the novel E-commerce platform.

Originality/value - The authors believe that the MCIN, first proposed in this paper, is a transformational innovation which facilitates the infrastructure of the future networked system of supply-demand relationship.

Keywords E-commerce, Cyber physics social system, Multi-agent, Supply-demand relationship

Paper type Research paper

\section{Introduction}

In a new round of global revolution in technology and industry, the fusion of internet and other domains has produced a strategical and holistic impact on the development of economic society in various countries of the world. New models, technologies and applications are jointly breeding a future networked system of supply-demand relationship. Compared to

(C) Jianping Shen, Yadong Huang and Yueting Chai. Published in the International Journal of Crowd Science. Published by Emerald Publishing. This article is published under the Creative Commons Attribution (CC BY 4.0) licence. Anyone may reproduce, distribute, translate and create derivative works of this article (for both commercial and non-commercial purposes), subject to full attribution to the original publication and authors. The full terms of this licence may be seen at http://creativecommons.org/licences/by/4.0/legalcode
Received 3 December 2016 Revised 29December 2016 13January 2017 Accepted 19January 2017 


\section{IJCS \\ 1,1}

traditional systems, the future networked system of supply-demand relationship has four essential characteristics: proactive personalized consumption, direct centralized distribution, intelligent decentralized manufacture and ecological self-organized system. To facilitate such characteristics, a novel network, which evolves from traditional internet to a large-scaled, open-styled, self-organized and ecological intelligent network, called material conscious information network (MCIN), is required by the aforementioned networked system.

The MCIN has essential logics of openness, federation, cooperation and sharing, and characteristics of consistency, efficiency, innovation and sustainability. In the MCIN, the nodes are information counterparts of person, enterprise, administrative department and thing in both material and conscious worlds. Any relationship between the MCIN nodes is a supply-demand relationship. From a behavioral perspective, interactions between any nodes of the MCIN are behaviors or activities of trading in some extent. Each node of the MCIN is facilitated with a set of intelligent federated agents which enable the nodes to perceive, think, interact and evolve adaptively.

Using a counterpart to reflect material-conscious individuals on information world mostly emerges in the research of cyber-physical system, e.g. Cyberself, Cyber-Individual, etc. Cyberself (Robinson, 2007) is proposed to be an embodiment of an individual as a means of identity signaling and as a medium of online interaction, whereas Cyber-Individual (Wen et al., 2009), with a short term as "Cyber-I", is the unique counterpart of a real person on cyberspace and also a digital counterpart going with one's whole life. However, studies on the counterpart of a realistic individual remain in the conceptual phase and is incapable to model the MCIN.

Modeling of the MCIN is a two-step process. In the first step, each node of the MCIN is modeled in a generic way. Second, an addressing approach for each node in the MCIN is defined. There are three main challenges in the modeling of the MCIN:

(1) how can we model the basic and supply-demand information of each kind of nodes generically?

(2) how can we build the architecture for the intelligent federated agents of each node to achieve supply-demand interactions spontaneously?

(3) how can we define a supply-demand-oriented addressing method for each node?

In our previous work, we proposed cyber-anima (Shen, 2015), a semantic model to reflect individuals in the cyberspace and reason their desires and intentions for recommender systems. Cyber-anima has six dimensions, namely, physiology, belief, character, knowledge, experience and context, which correspond to the intrinsic and extrinsic factors of humanity. On the one hand, cyber-anima possesses the generalization which can develop to various disciplines, e.g. biology, medical science, psychology, sociology, statistics, economics, management science, politics, philosophy, anthropology, etc. On the other hand, a fourunitary semantic structure of dimension, concept, relation and axiom empowers cyberanima to reflect any realistic participants to cyberspace. Based on cyber-anima, an animadesire-intention-based (ADI-based) reasoning approach is proposed for recommendation interactions, which can also be extended to the scenario of trading-styled interactions.

In this work, we present an approach based on cyber-anima to model the MCIN. Leveraging and refining the dimensions of structure, character, knowledge and experience, we extend cyber-anima to fit the basic information of all kinds of the MCIN nodes, e.g. persons, enterprises, departments of government and thing, and present a double-chain structure for both basic and supply-demand information of each node. We propose an ADIbased architecture for the intelligent federated agents of each node in the MCIN. We propose a six-degrees-of-separation blockchain for the MCIN node addressing. We develop a 
prototype of novel E-commerce platform based on the MCIN to prove the effectiveness and soundness of the MCIN modeling.

The main contributions of this paper are as follows:

- design a generic model for the basic and supply-demand information of the MCIN nodes based on cyber-anima;

- propose an ADI-based multi-agent architecture for the MCIN nodes to drive their spontaneous trading-styled interactions; and

- present a novel node addressing method based on six-degrees-of-separation blockchain for the MCIN.

The rest of the paper is organized as follows: Section 2 discusses existing related work and highlights the key contributions of this study of work. Section 3 provides a detailed elucidation of the generic model for the MCIN nodes. Section 4 focuses on the multi-agent architecture of the MCIN nodes. Section 5 presents the node addressing method in the MCIN. Section 6 develops a prototype system based on the modeled MCIN and discusses its efficiency. Section 7 concludes the work and provides a summary of future work.

\section{Literature review}

The research on cyber-physical system has been quite popular recently. Using counterparts to reflect realistic individuals is the first step to modeling a cyber-physical system. To this end, several concepts have been proposed. Robinson proposed an embodiment of individual as a means of identity signaling and as a medium of online interaction, called Cyberself (Robinson, 2007). Besides Cyberself, Robinson also presents Cyberpersona and Cyberbody as the counterparts of persona and body in cyberspace. Wen et al. proposes Cyber-Individual, with a short term "Cyber-I", to create a unique, digital, comprehensive description for every real person existing in the cyberspace including human's social context, mood, temper, physical status and so on (Wen et al., 2009). Cyber-I is a real individual's counterpart in the cyberspace. However, research of this kind is still in its conceptual phase and cannot be applied to the modeling of the cyber-physical system directly.

Eskins et al. presents a framework of modeling Cyber-Human Systems (CHS) which divides CHS elements into four types: components, participants, processes and tasks (Eskins et al., 2001). Each type of CHS elements is related to a set of properties. The framework of modeling CHS provides a structured and quantitative method of analyzing cyber security problems whose outcomes are influenced by human-system interactions. Hence, such a framework can only be applied to a specific domain and its modeling of CHS lacks of generality and semantization.

In our previous work, we proposed a semantic model, called cyber-anima, to reflect individuals in the cyberspace and reason their desires and intentions for recommender systems (Shen, 2015). Cyber-anima composing of physiology, belief, character, knowledge, experience and context represents the intrinsic and extrinsic factors of user humanity. We performed a study on the semantic modeling approach based on the cyber-anima and proposed a multi-phase, multi-task and multi-technology mechanism for learning and populating the terms, concepts, taxonomy relations, non-taxonomy relations and axioms in the cyber-anima. We presented the cyber-anima-based reasoning approaches to derive individual user desire and intention, which leverage the existing or past individual desires and individual intentions with a template to reason over with. The cyber-anima can produce individual desire-oriented user profile and individual intention-oriented recommendation based on cyber-anima model. Albeit cyber-anima was proposed for enhancing the 
IJCS

1,1

performance of the recommender system. Its generic and semantic modeling approach, model learning mechanism and ADI-based reasoning framework can be extended to model the novel network of supply-demand relationships, especially for the MCIN.

\section{A cyber-anima-based model of the material conscious information network node}

The first main challenge in modeling of the MCIN is: how can we model the basic and supply-demand information of each kind of nodes generically? In this section, we introduce a generic model for both basic and supply-demand information of the MCIN nodes based on cyber-anima.

\subsection{Model the basic information}

The MCIN is a large-scaled, open-styled, self-organized and ecological intelligent network. The nodes of the MCIN are information counterparts of supply and demand sides in realistic trading activities or interactions, which can be divided into four categories: person, enterprise, administrative department and thing. Each of the MCIN node has basic and supply-demand information. The basic information reflects the material world of a node, whereas the supply-demand information represents the history of the conscious world of a node.

In the light of the aforementioned characteristics, a model which can generically represent the basic information of all four categories of the MCIN nodes should be designed from a cross-disciplinary perspective. In particular, each category of the MCIN nodes relates to both general and specific disciplines, shown in Table I.

In our previous work (Shen, 2015), we proposed a semantic model, called cyber-anima, to reflect individuals in the cyberspace and reason their desires and intentions for recommender systems. In light of the equivalence between "individual" and "person" in our previous work and this work, respectively, the basic information of the MCIN person node can be modeled by cyber-anima. Without the loss of generality, we formalize the basic information of The MCIN nodes based on cyber-anima as tuple $I_{B}$ with four attributes, as explained below.

Definition 1 (Model of the basic information of the MCIN node). The basic information of the MCIN node is modeled based on cyber-anima as tuple $I_{B}$ with four attributes:

$$
I_{B}::=<D_{S T}, D_{C H}, D_{E X}, D_{K N}>
$$

where:

$D_{S T}$ is the structure dimension;

$D_{C H}$ is the character dimension;

$D_{E X}$ is the experience dimension; and

$D_{K N}$ is the knowledge dimension.

\begin{tabular}{|c|c|c|}
\hline Category & General discipline & Specific discipline \\
\hline Person & \multirow{4}{*}{$\begin{array}{l}\text { Economics, natural } \\
\text { science }\end{array}$} & Biology, medical science, psychology, sociology, anthropology \\
\hline Enterprise & & $\begin{array}{l}\text { Organizational behavior, management science, operations } \\
\text { research, statistics, sociology, anthropology }\end{array}$ \\
\hline $\begin{array}{l}\text { Administrative } \\
\text { department }\end{array}$ & & $\begin{array}{l}\text { Public administration, politics, management science, statistics, } \\
\text { sociology, anthropology }\end{array}$ \\
\hline Thing & & Engineering science, design science, statistics \\
\hline
\end{tabular}

\section{Table I.}

Category of the MCIN participant and its related disciplines 
In accordance with Definition 1, the dimensions of the basic information of the MCIN person node are designed with cross-disciplinary perspective shown in Table II. The basic information of the MCIN person node has four dimensions including structure, character, experience and knowledge.

For the sake of self-organized, the basic information of The MCIN nodes are modeled semantically. Dimension is the maximum granularity composition of the semantic model. Formally, we define a dimension of the basic information of the MCIN nodes as tuple $D$ with two attributes, as explained below.

Definition 2 (Dimension). A dimension of the basic information of the MCIN node is modeled as tuple $D$ with two attributes:

$$
D::=<C, R>
$$

where:

$C$ is the set of concepts, and $C::=\langle M C, A X C>$, where $M C$ is the marker of concept, and $A X C$ is the set of concept axioms; and

$R$ is the set of relations, and $R::=<M R, C P, A X R>$, where $M R$ is the marker of relation, $C P$ is the pair of concept, and $A X R$ is the set of relation axioms.

To clarify the representation of dimensions, we give each dimension several classifications to distinguish their concepts with different perspectives. For example, the structure dimension of the basic information of the MCIN person node has two classifications of DNA and physique distinguished in perspectives of biology and medical science, respectively. Accordingly, we can extend the MCIN person node to model the other three categories of the MCIN nodes with identical dimensions but different classifications of concept. Tables III-V show the dimensions of the basic information of the MCIN enterprise node, the MCIN administrative department node and the MCIN thing node with their classifications and perspectives.

Based on cyber-anima, the basic information of all four categories of the MCIN nodes has been modeled generically and semantically. In maximum granularity level, such models

\begin{tabular}{lll}
\hline Dimension & Classification & Perspective \\
\hline Structure $\left(\mathrm{D}_{\mathrm{ST}}\right)$ & DNA, physique & Biology, medical science, natural science \\
Character $\left(\mathrm{D}_{\mathrm{CH}}\right)$ & 16PF, Big Five, psychogenic needs & Psychology, sociology \\
Experience $\left(\mathrm{D}_{\mathrm{EX}}\right)$ & Life, work, study, trade & Psychology, sociology \\
Knowledge $\left(\mathrm{D}_{\mathrm{KN}}\right)$ & Discipline, skill & Sociology, psychology
\end{tabular}

Table II. Dimensions of the basic information of the MCIN person node

\begin{tabular}{lll}
\hline Dimension & Classification & Perspective \\
\hline Structure $\left(\mathrm{D}_{\mathrm{ST}}\right)$ & $\begin{array}{l}\text { Finance, ownership, } \\
\text { organizational structure } \\
\text { Brand, corporate culture }\end{array}$ & $\begin{array}{l}\text { Organizational behavior, economics, management } \\
\text { science, statistics, natural science } \\
\text { Organizational behavior, management science, } \\
\text { sociology, philosophy }\end{array}$ \\
Experience $\left(\mathrm{D}_{\mathrm{CH}}\right)$ & $\begin{array}{l}\text { R\&D, marketing, management, } \\
\text { production, trade }\end{array}$ & $\begin{array}{l}\text { Organizational behavior, management science, } \\
\text { operations research, sociology } \\
\text { Knowledge }\left(\mathrm{D}_{\mathrm{KN}}\right)\end{array}$ \\
$\begin{array}{l}\text { Production management, } \\
\text { operation }\end{array}$ & $\begin{array}{l}\text { Ecomics, management science, operations } \\
\text { research, statistics }\end{array}$ \\
\hline
\end{tabular}

Table III.

Dimensions of the basic information of the MCIN enterprise node 
IJCS

1,1

Table IV.

Dimensions of the basic information of the MCIN administrative department node have uniform dimensions: structure, character, experience and knowledge. Albeit the dimensions of the basic information of the MCIN nodes are identical in semantic structure, they are distinguished by different classifications generated in different perspectives. We use these classifications as an explanatory tool, so these classifications of dimensions illustrated above are not exclusive and varied with weights of blended perspectives.

\subsection{Construct information for the material conscious information network node}

The basic information of The MCIN nodes has been generically and semantically modeled with four dimensions including structure, character, experience and knowledge. As we all know, anything in the material world will be changed by the environment eventually with the time. The dimensions of the basic information of the MCIN nodes have to be synchronized with these changes. As aforementioned, the history of the conscious world of the MCIN node can be represented by its supply-demand information. In a temporal perspective, the two worlds of a the MCIN node interact as both cause and effect. For the sake of modeling, the MCIN nodes, both basic and supply-demand information have to be constructed in a temporal chain styled formation. Formally, we define the model of the MCIN nodes as tuple $\mathcal{A}$ with four attributes, as explained below.

Definition 3 (Model of the MCIN node). An MCIN node is modeled based on cyber-anima as tuple $\mathcal{A}$ with four attributes:

$$
\mathcal{A}::=<I_{B}, I_{S}, I_{D}, T>
$$

where:

$I_{B}$ is the basic information of a MCIN node;

$I_{S}$ is the supply information of a MCIN node;

$I_{D^{-}}$is the demand information of a MCIN node; and

$T$ is the set of time points.

\begin{tabular}{lll}
\hline Dimension & Classification & Perspective \\
\hline Structure $\left(\mathrm{D}_{\mathrm{ST}}\right)$ & Finance, system & $\begin{array}{l}\text { Public administration, politics, management } \\
\text { science, statistics, natural science }\end{array}$ \\
Character $\left(\mathrm{D}_{\mathrm{CH}}\right)$ & $\begin{array}{l}\text { Style of work, administrative } \\
\text { culture }\end{array}$ & $\begin{array}{l}\text { Public administration, politics, management } \\
\text { science, sociology }\end{array}$ \\
Experience $\left(\mathrm{D}_{\mathrm{EX}}\right)$ & $\begin{array}{l}\text { Administrative affair, } \\
\text { function adjustment, trade } \\
\text { Administration, service, } \\
\text { Knowledge }\left(\mathrm{D}_{\mathrm{KN}}\right)\end{array}$ & $\begin{array}{l}\text { Public administration, politics, economics, } \\
\text { management science }\end{array}$ \\
\hline
\end{tabular}

Table V.

Dimensions of the basic information of the MCIN thing node

\begin{tabular}{lll}
\hline Dimension & Classification & Perspective \\
\hline Structure $\left(\mathrm{D}_{\mathrm{ST}}\right)$ & Material, composition & $\begin{array}{l}\text { Natural science, engineering science, design science, } \\
\text { statistics }\end{array}$ \\
Character $\left(\mathrm{D}_{\mathrm{CH}}\right)$ & $\begin{array}{l}\text { Function, quality, size, } \\
\text { manufacture cost }\end{array}$ & $\begin{array}{l}\text { Economics, natural science, engineering science, } \\
\text { design science, statistics }\end{array}$ \\
Experience $\left(\mathrm{D}_{\mathrm{EX}}\right)$ & $\begin{array}{l}\text { Design, manufacture, } \\
\text { logistics, install }\end{array}$ & $\begin{array}{l}\text { Economics, engineering science, design science, } \\
\text { statistics }\end{array}$ \\
Knowledge $\left(\mathrm{D}_{\mathrm{KN}}\right)$ & Technology, patent & Natural science, engineering science, design science \\
\hline
\end{tabular}


The model of the MCIN node has a DNA-styled double-chain structure. As shown in Figure 1, there are two chains in the model which represent the supply information and demand information, respectively. The connector of these two chains stands for the basic information of the MCIN node. The whole chain is growing with time, and each minimum segment of the double chain refers to the basic and supply-demand information of the MCIN nodes in a particular time point.

\section{An anima-desire-intention-based multi-agent architecture for the material conscious information network nodes}

The second main challenge in modeling of the MCIN is: how can we build the architecture for the intelligent federated agents of each node to achieve supply-demand interactions spontaneously? After modeling the MCIN nodes based on cyber-anima, we explore the architecture of a multi-agent for the MCIN nodes to enable the spontaneous interactions in this section.

\subsection{Spontaneous interactions required for the material conscious information network nodes}

The MCIN is a ternary system of material, conscious and information, which networks any supply-demand relationships. Figure 2 illustrates the interactions between the MCIN nodes which are in a trading style. Services and information are demanded and supplied by the MCIN nodes mutually.

No matter which kind of the MCIN nodes, they have the same pattern of interactions including creating supply-demand information, searching supply-demand information, conforming supply-demand information, delivering service, searching particular nodes, linking with other nodes, etc. Though persons, enterprises, administrative departments and things can interact among themselves, most of such time-consuming work has better been efficiently accomplished by intelligent federated agents facilitated by the MCIN nodes.

These intelligent federated agents perceive, think, interact and evolve as a whole through cooperating and collaborating. Each agent has its particular intelligence and generates outputs by handling different inputs. As an example, some of the agents can handle vision (graphics), auditory (audio) and touch (pressure, vibration, temperature

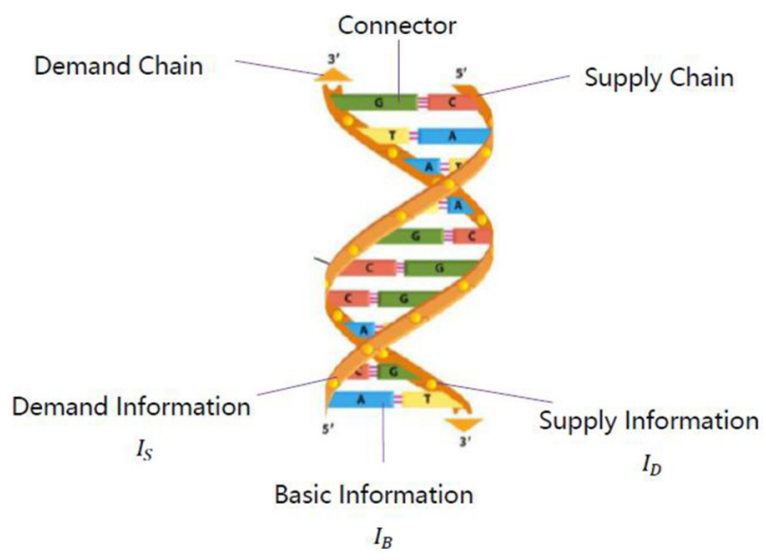

Figure 1. DNA-styled doublechain structure of the MCIN node 


\section{IJCS \\ 1,1}

16

\section{Figure 2.}

Interactions between the MCIN nodes



and humidity) inputs and generate manageable outputs, and others can think of the subsequent reactions toward such outputs. Different MCIN nodes have different levels of intelligence on account of its agents and can evolve to a higher level via interacting mutually.

\subsection{Build anima-desire-intention-based multi-agent architecture}

For the purpose of perceiving, thinking, behaving and evolving as a whole, the federated agents of the MCIN nodes require an efficient multi-agent architecture to self-organize. In our previous work (Shen, 2015), we proposed an ADI-based multi-agent architecture for recommender system. As intelligent interactions between the MCIN nodes have a similar scenario of recommending items between users, we can extend and use the architecture for the agents of the MCIN nodes. To this end, we formalize the spontaneous interaction generation problem as follows:

Definition 4 (Spontaneous interaction generation problem). For each MCIN node, $n \in N$ has a desire $d_{t_{i}}^{w} \in \mathcal{D}_{n}$ and an interaction $i_{t_{i}}^{w} \in \mathcal{I}_{n}$ on time point $t_{\mathrm{i}} \in T$ in possible world $w \in W$ according to its Cyber - Anima $\mathcal{A}_{n}$, we want to choose such interaction $\boldsymbol{i}_{t_{i}}^{w}$ that $i_{t_{i}}^{w}=\delta\left(\right.$ maxexpval, $\left.d_{t_{i}}^{w}\right)$.

where:

$W$ is a set of possible worlds, and for each $w \in W$, we have $w::=<T_{w}, A_{w}, E_{w}>$. $T_{w} \subseteq T$ is a set of time points in possible world $w$; $A_{w}$ is a binary relation of time points, namely, $\forall i\left(t_{i}, t_{i+1}\right) \in A_{w} ; E_{w}$ is a set of event type in possible world w;

$\mathcal{D}_{n}$ is the possible desire world of The MCIN nodes $n \in N$, and $\mathcal{D}_{n} \subseteq W \times T \times W$; $\mathcal{I}_{n}$ is the possible interaction world of The MCIN nodes $n \in N$, and $\mathcal{I}_{n} \subseteq W \times T \times W$; $\delta$ is the deliberation function;

$\mathcal{A}_{n}$ is the Cyber-Anima model of The MCIN nodes $n \in N$; and maxexpval is the maximizing expected value. 
In light of Definition 4, generating an optimum interaction for a particular MCIN node in a specific time point is a twofold process shown in Figure 3. First, we reason the desire $d_{t_{i}}^{w}$ of the MCIN node based on its cyber-anima model. Second, we reason and find such an interaction $i_{t_{i}}^{w}$ that $i_{t_{i}}^{w}=\delta\left(\right.$ maxexpval, $\left.d_{t_{i}}^{w}\right)$.

For the purpose of reasoning the desire of the MCIN node, we acquire the model of the MCIN node model $\mathcal{A}_{n}$ on current time point $t_{i}$, then drive the agents for perceiving and thinking to reason each event type $e_{t_{i}}^{w}$ of node's desire $d_{t_{i}}^{w}$ along with its payoff value $\operatorname{PAYOFF}\left(e_{t_{i}}^{w}\right)$ on current time point $t_{i}$ based on the MCIN node model $\mathcal{A}_{p}$. The node's desire $d_{t_{i}}^{w}$ illustrates who it expects to interact with and what service or information it expects to interact for. Next, we drive the agents for perceiving and thinking again to reason the node's interaction $i_{t_{i}}^{w}$ which illustrates the probability $\operatorname{PROB}\left(e_{t_{i}}^{w}\right)$ and payoff value $\operatorname{PA} \operatorname{YOFF}\left(e_{t_{i}}^{w}\right)$ toward each candidate to be interacted on current time point $t_{i}$ based on node's desire $d_{t_{i}}^{w}$. Finally, we sum up the utility value $\operatorname{PAYOFF}\left(e_{t_{i}}^{w}\right) \cdot \operatorname{PROB}\left(e_{t_{i}}^{w}\right)$ of each candidate in the node's interaction $i_{t_{i}}^{w}$ and sort for the optimal interactions. As the model, desire and interaction of the MCIN node are built on material world, conscious world and information world, respectively, the process of generating spontaneous interaction based on ADI reasoning is an exploration from unary world to ternary world via binary world.

Furthermore, the process of evolving intelligence level of The MCIN nodes is just a remix of $\mathrm{ADI}$ reasoning process, namely $\mathrm{ADI}$ evolving. In ADI evolving, we can explore from ternary world to unary world via binary world, to train the agents with given sets of model, desire and interaction of the MCIN node.

\section{An addressing method based on six-degrees-of-separation blockchain}

The last main challenge in modeling of the MCIN is: how can we define a supply-demand oriented addressing method for each node? As modeling of single MCIN nodes has been discussed, this section explains the networking of the MCIN nodes and addressing method in the MCIN.

\subsection{Build the material conscious information network based on six-degrees-of-separation blockchain}

As a future networked system of supply-demand relationship, the MCIN has the characteristics of coevolving intelligence, decentralized organization and efficient addressing. Coevolving intelligence relies on the multi-agent architecture of the intelligent federated agents of the MCIN nodes, which has been discussed in the last sections. Once an MCIN node is created, it would like to link to other nodes, evolve constantly and never destroy. For the purpose of linking, these MCIN nodes together and building the decentralized network, we propose a six-degrees-of-separation blockchain.

\section{Information World}
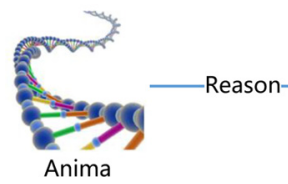

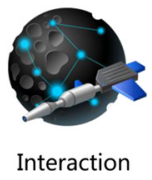

Material conscious information network 
IJCS

1,1

Blockchain (Nakamoto, 2008) is a kind of distributed database, which serves as the public ledger for all transactions of Bitcoin, a decentralized digital currency, after conceptualization. We use blockchain to enable the decentralization of the MCIN and make the following specifications of the MCIN blockchain:

- each MCIN person, enterprise or administrative department node must join a blockchain with at least $\mathrm{N}$ different The MCIN nodes, where $N=$ $[\sqrt[5]{\text { total amount of MCIN person, enterprise and administrative department nodes }}]$;

- each MCIN thing node must join a blockchain with at least five different the MCIN nodes; and

- each MCIN blockchain must have at least one MCIN administrative department node.

The joining of the MCIN blockchain is driven by a supply-demand relationship. Whenever the MCIN nodes construct new supply-demand relationships with other the MCIN nodes, they may join or create new MCIN blockchain. As one of the interactions, the selection of blockchain is accomplished by the agents of the MCIN nodes spontaneously according to abovementioned specifications. Therefore, in each MCIN blockchain, we have the following truths:

- each member of the blockchain has a supply-demand relationship with at least one other member;

- all members of a blockchain maintain an equilibrium of supply-demand relationship; once the equilibrium is broken, new members may join the blockchain; otherwise, the old members may join or create new blockchain; and

- via at most six MCIN blockchains, any two MCIN nodes can link with each other. It means that the MCIN blockchain satisfies six-degrees-of-seperation (Milgram, 1967).

Leveraging the MCIN blockchain, each MCIN node can acquire a unique, immutable address. Formally, we define the address of the MCIN nodes as follows:

Definition 5 (the MCIN blockchain and node address). For each MCIN blockchain $b c \in B C$ has a member set of the MCIN nodes $N$. Then, we have $A D D R_{b c}=$ $\operatorname{Hash}\left(I_{B_{1}}, I_{B_{2}}, I_{B_{3}}, \ldots, I_{B_{n}}\right)$. For each MCIN node $n \in N$ in the MCIN blockchain $b c$, we have $A D D R_{n}=A D D R_{b c}+\operatorname{Hash}\left(I_{B_{n}}\right)$.

where:

$I_{B}$ is the basic information of a MCIN node;

$A D D R_{b c}$ is the address of the MCIN blockchain $b c \in B C$;

$A D D R_{n}$ is the address of the MCIN nodes $n \in N$; and

Hash is the hash function to generate the address code.

Figure 4 illustrates the process of generating the MCIN blockchain and node address. As an example, China government, Beijing city government and Haidian district government are the MCIN administrative department nodes, which are linked to the same MCIN blockchain. In the light of Definition 5, the address of the MCIN blockchain can be generated by hashing all basic information of these three MCIN administrative department nodes, which is a unique, immutable address. Meanwhile, the address of a single MCIN node, e.g. China government, can be generated by hashing its basic information concatenating the address of the MCIN blockchain. 


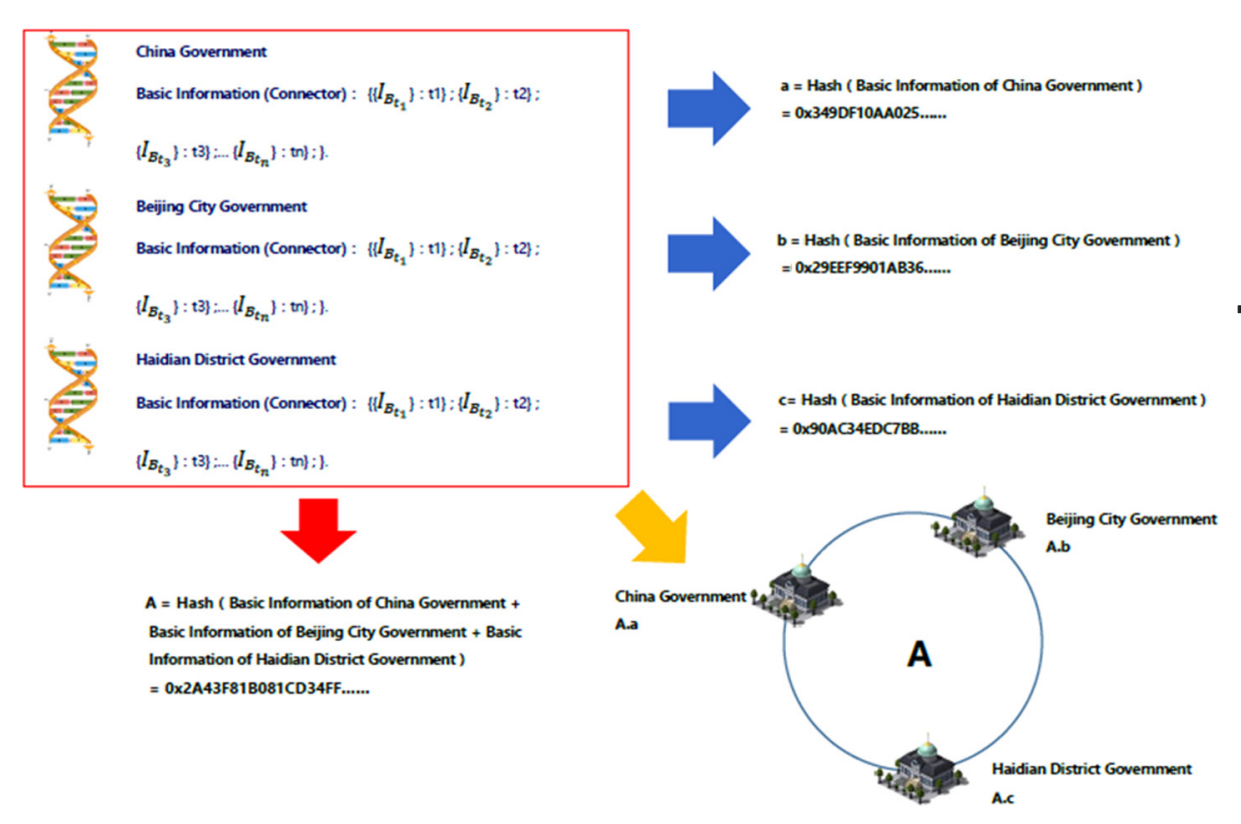

\section{Material conscious information network}

Figure 4.

Process of generating the MCIN blockchain and node address

\subsection{Supply-demand-oriented addressing}

As a decentralized network, the MCIN requires an efficient addressing method. Compared to a centralized network, the addressing method of the MCIN has the characteristics of socialization, information integration, collective intelligence, traceability, high robustness, unification of producing and consuming, high scalability and decentralization. As the MCIN is a future networked system of supply-demand relationship, we propose a supplydemand-oriented addressing method to locate target nodes efficiently via supply-demand relationships.

In light of Definition 3, the MCIN nodes are modeled with supply and demand chains, i.e. the supply information $I_{S}$ and the demand information $I_{D}$, which represent the history of supply and demand respectively. The supply and demand information is materialization of conscious world of the MCIN nodes and can be modeled in the same way. Formally, we define the model of the supply and demand information as tuple with two attributes, as explained below:

Definition 6 (Model of the supply and demand information of the MCIN node). The supply and demand information of the MCIN nodes are modeled as tuple $I_{S}$ and $I_{D}$ with two attributes:

$$
I_{S}=I_{D}::=<R, P>
$$

where:

$R$ is a set of supply and demand request, we have $R::=\left\langle C_{\text {object }}, C_{\text {attribute }}\right\rangle$, where $C_{\text {object }}$ is the set of concepts of request object, and $C_{\text {attribute }}$ is the set of concepts of the attributes correspondent to the object; and

$P$ is a set of addressing path correspondent to the request. For each addressing path $p \in P$, we have the partially ordered set $p=\left(A D D R_{b c}, \ldots, A D D R_{n}\right)$. 
IJCS

1,1

20

The request of the MCIN node addressing is a segment of the supply and demand information. The supply-demand-oriented addressing method begins from the MCIN node of requester and traverses a certain number (not all) of blockchains to locate the MCIN nodes which match the request. Leveraging the supply and demand information of the MCIN nodes, the performance of addressing can be improved with the amount of similar requests. Figure 5 illustrates an example of supply-demand-oriented addressing in the MCIN. In Figure 5(a), Jerry launches a request, and the addressing of his request first starts in his directly linked blockchain A to find matched requests in supply and demand information. Unfortunately, no such requests matches. Then, in Figure 5(b), the addressing extends to adjacent blockchain A and B via Haidian District Government. This time, a matched supply request is found in the supply information of Supermarket. As Jerry and Supermarket have a supply-demand relationship after the addressing, a new blockchain D is created, and the path of Jerry's request is from Jerry to Supermarket via blockchain D. In Figure 5(c), Angela launches a similar request, and the addressing of her request first starts in blockchain A as well. Obviously, Jerry's demand request matches. Then in Figure 5(d), a matched supply request is found in the supply information of Supermarket by leveraging the path of Jerry's previous demand request.

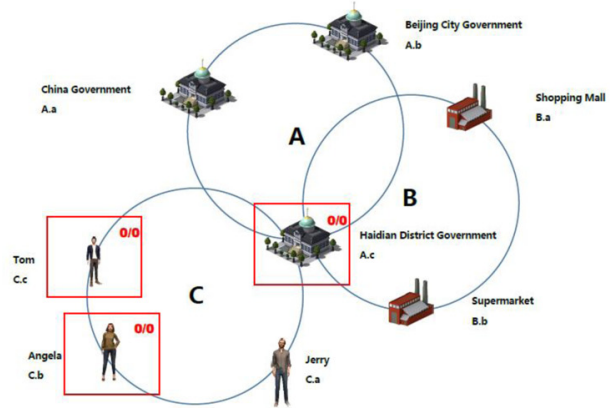

(a)

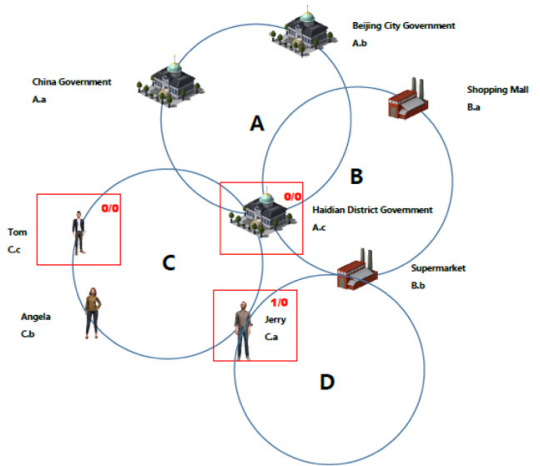

(c)

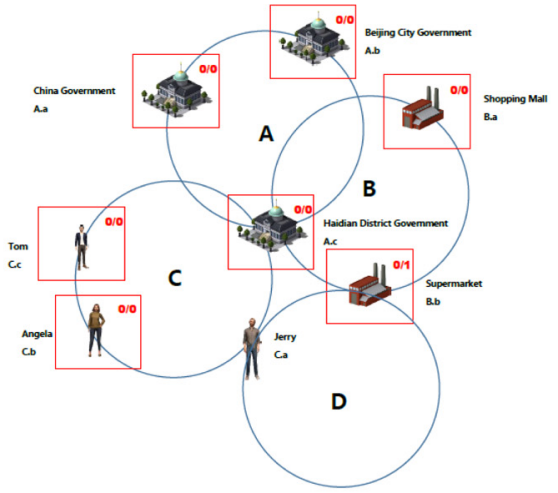

(b)

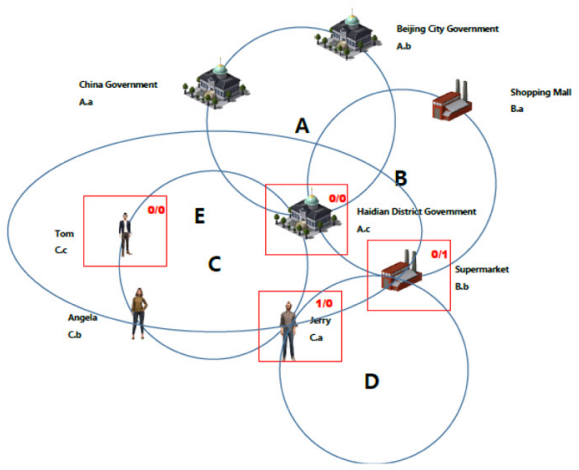

(d)
Figure 5.

Process of supplydemand-oriented addressing

\section{(c)}


As Angela and Supermarket have a supply-demand relationship after the addressing, a new blockchain E is created, and the path of Angela's request is from Angela to Supermarket via blockchain E. The first addressing traverses 3 MCIN blockchains and 7 MCIN nodes, whereas the second addressing for the similar request traverses 1 MCIN blockchain and 4 MCIN nodes. With the supply-demand-oriented addressing method, the same type of similar requests in others' supply-demand information can efficiently improve the addressing performance.

\section{Prototype implementation and discussion}

The MCIN is proposed for the purpose of implementing the future networked system of supply-demand relationship. Compared to traditional systems, the future networked system of supply-demand relationship has four essential characteristics: proactive personalized consumption, direct centralized distribution, intelligent decentralized manufacture and ecological self-organized system. The future networked system of supply-demand relationship has to build its own operation platform, a novel E-commerce platform.

In the last sections, we have generically modeled the MCIN nodes based on cyber-anima, presented an ADI-based multi-agent architecture for intelligent federated agents of the MCIN nodes and proposed an addressing method for the MCIN. In this section, we implement the prototype system of the novel E-commerce platform relied on the modeling of the MCIN. The prototype system composes of four function modules: Who am I; My demand; My supply; and My space.

The novel E-commerce platform is a personalized portal for every unique MCIN node, which means that any the MCIN node has its own independent portal. Intuitively, we call each MCIN node the owner of its platform. The model of the MCIN node drives the platform for the information counterpart of its owner in the MCIN autonomously, and meanwhile, the owner can operate the platform manually. The platform shown in Figure 6 shows the 3D virtual image of its owner and entrances of the four function modules at a first glance.

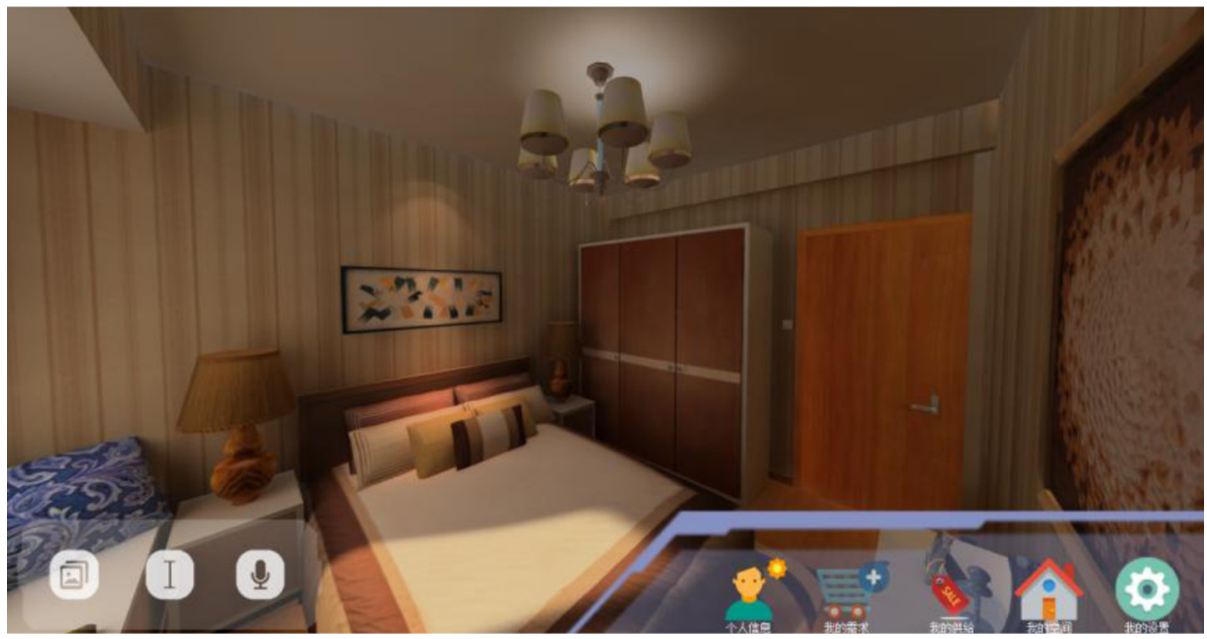

Figure 6.

The novel e-commerce platform at a first glance 
IJCS

1,1

22

The "Who am I" function module is a snapshot of the owner's model of the MCIN node. In this function module, we can overview interested basic and supply-demand information of owner's model of the MCIN node to know who it is. Figure 7 shows the "Who am I" function module of the MCIN person node, including her 3D virtual image, physique-related concepts of structure dimension, values-related concepts of character dimension, trade-related concepts of experience dimension, etc.

The "My demand" function module tackles all demands of its owner rely on owner's model of the MCIN node and supply-demand-oriented addressing method, covering pullstyled demand and push-styled demand. Any interaction in the MCIN is trade-styled and associated with a demand part and a supply part, so the owner is a demand part in "My demand" function module. The owner's demand can proactively be launched by itself or passively be elicited by the supply side, which are so-called pull-styled and push-styled, respectively. No matter who launches the demand, an optimal matching of demand and supply sides can be generated by the supply-demand oriented addressing. Figure 8 illustrates the supply-demand oriented addressing snapshot for a pull-styled demand of automobile.

Relatively, "My supply" function module handles all supplies of its owner relying on an owner's model of the MCIN node and the supply-demand oriented addressing method, covering pull-styled supply and push-styled supply. As the supply part in the "My supply" function module, the owner's supply can proactively be launched by itself or passively be elicited by the demand side, which are so-called pull-styled and push-styled respectively. No matter who launches the supply, an optimal matching of demand and supply sides can be generated by supply-demand-oriented addressing. Figure 9 shows the supply interface.

The "My space" function module is a visualization of the MCIN from the owners' perspective. Figure 10 presents a snapshot of a "My space" function module. The node in the center represents the owner of the platform, while other nodes around represent persons, enterprises, administrative departments and things directly and indirectly linking to the owner via six-degrees-of-separation blockchain. Driven by the owner's model of the MCIN node and the supply-demand oriented addressing method, the owner's MCIN node would be linked in more and more six-degrees-of-separation blockchain.

Figure 7.

"Who am I" function module

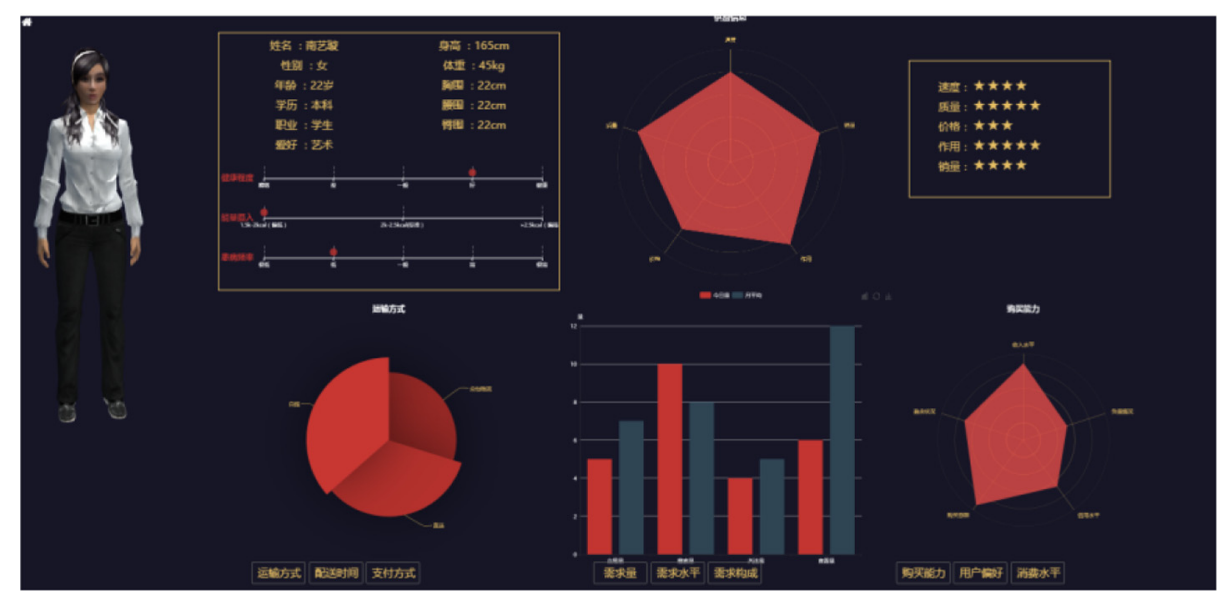




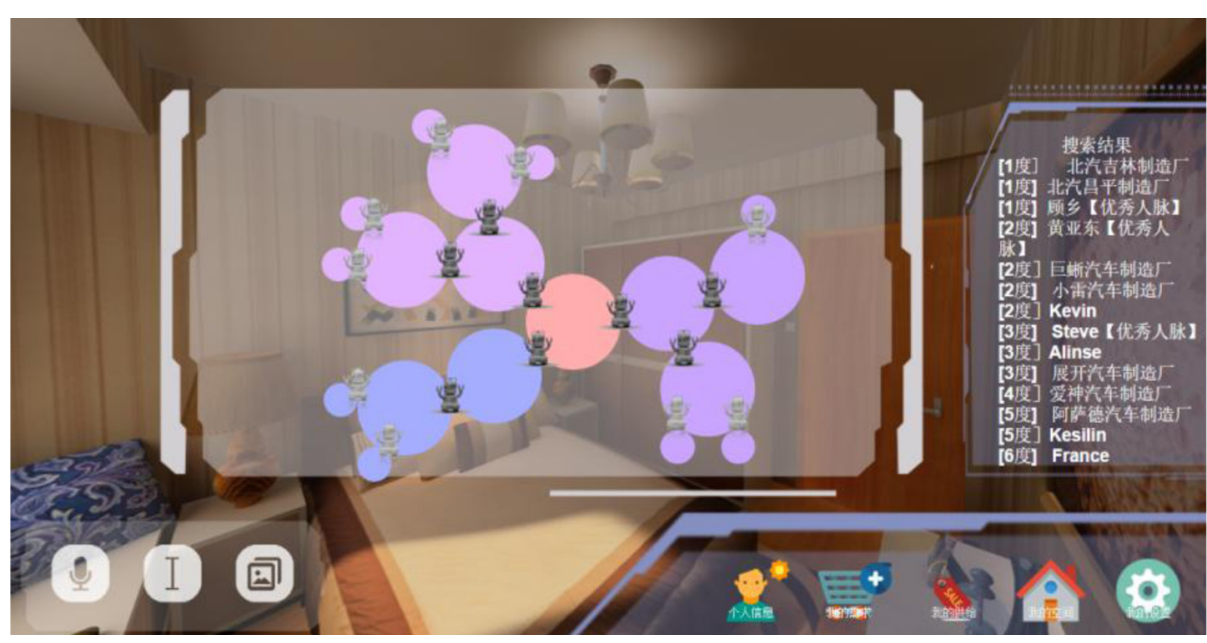

\section{Material conscious information network}

23

Figure 8.

"My demand" function module

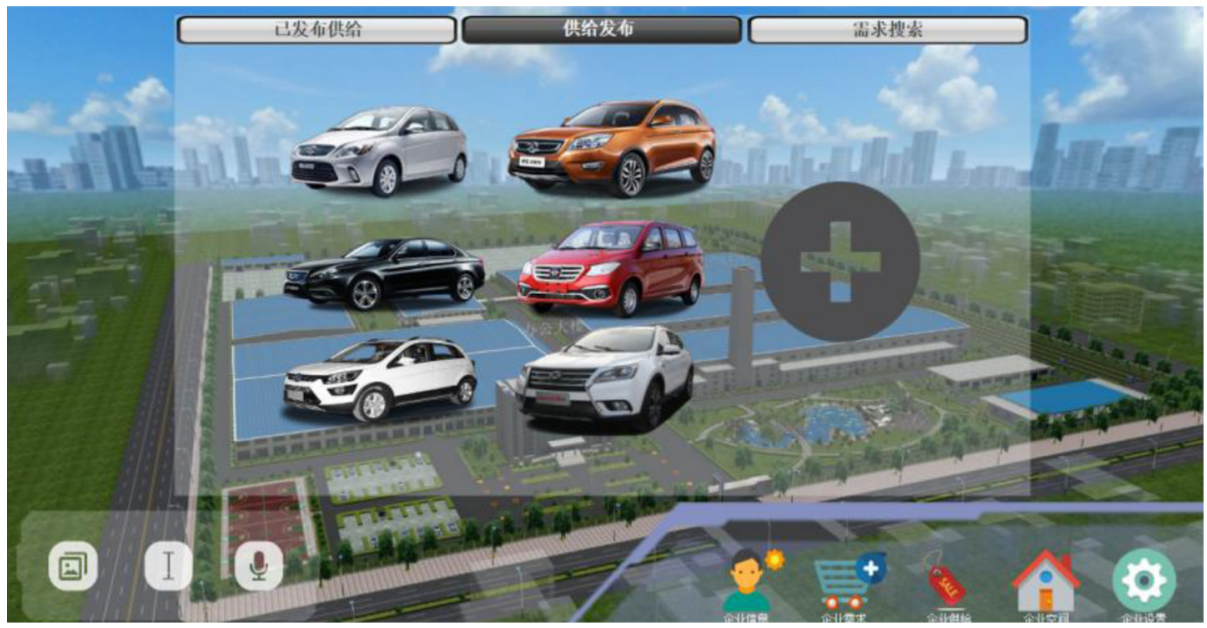

Figure 9.

"My supply" function module

Compared to traditional ones, the novel E-commerce platform enhanced by the MCIN has the advantages in:

- directly connection with consumer end and producer end directly;

- intelligent trade matching; and

- personalized portal.

The modeling of nodes based on cyber-anima, the multi-agent architecture based on ADI and the addressing method oriented to supply and demand make the main differences between the MCIN and current internet, as shown in Table VI. 


\section{IJCS \\ 1,1}

Figure 10.

"My space" function module
Table VI.

Comparison between the MCIN and current internet

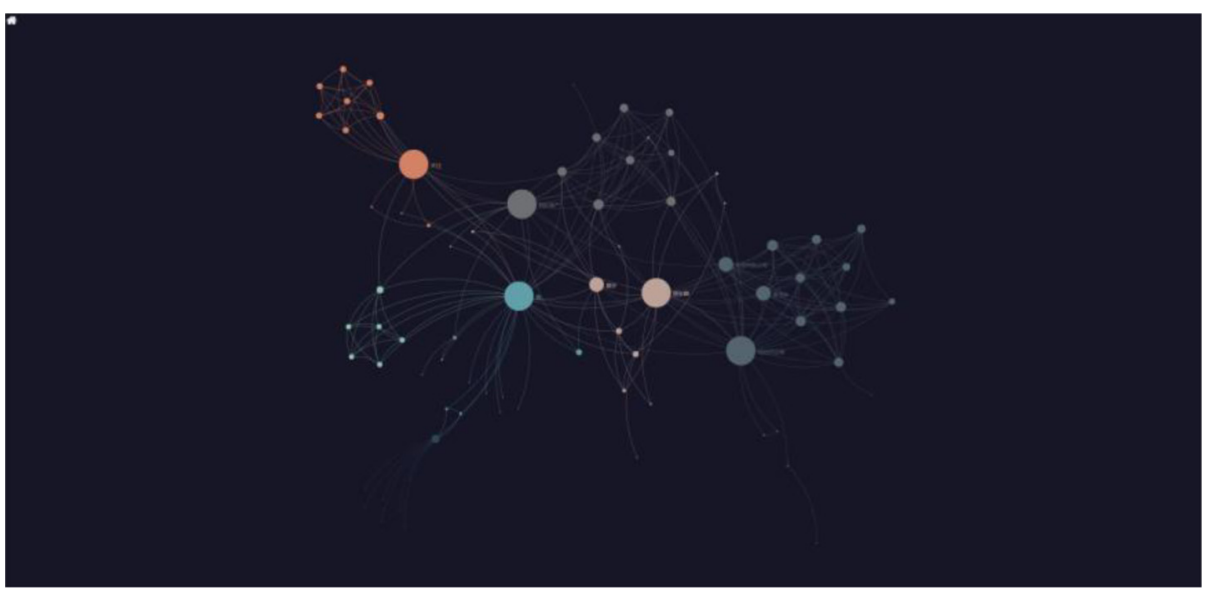

Current internet

Decentralization: information totally distributed in nodes

Socialization: searching rely on social relationship

Information integration: information of nodes can completely open

Collective intelligence: all nodes have intelligence and coevolve together

Traceability: able to trace to particular node High robustness: low negative influence when several nodes down

Unification of producing and consuming: nodes are not only producer but also consumer

High scalability: search cost is insensitive to the amount of nodes
Centralization: the process of collecting and handling information highly centralized

Non-socialization: searching along pages

Information fragmentary: users have to hide some critical information

Few intelligence: few search engines have intelligence and never coevolve

Non-traceability: information source suppressible Low robustness: completely stop working when search engine crashes

Opposition of producing and consuming: search engine produces results and users consume them Low scalability: search cost remarkably increase with the amount of nodes

\section{Conclusion and future work}

The MCIN is defined to be a large-scaled, open-styled, self-organized and ecological intelligent network, which evolves to the next generation of internet. The MCIN nodes are information counterparts of person, enterprise, administrative department and thing in both material and conscious worlds. Interactions between any MCIN node are trading-styled interactions to some extent. Each MCIN node equips a set of intelligent federated agents which enable the nodes perceive, think, interact and evolve adaptively. To model the MCIN, we have several main challenges to tackle.

To this end, we present an approach based on cyber-anima to model the MCIN. We extend the cyber-anima model with the dimensions of structure, character, knowledge and experience to fit the basic information of all kinds of the MCIN nodes, e.g. persons, enterprises, departments of government and thing. We present a double-chain structure for both basic and supply-demand information of each node. We propose an ADI-based 
architecture for the intelligent federated agents of each node in the MCIN. We present a sixdegrees-of-separation blockchain for the MCIN node addressing. We develop a prototype of novel E-commerce platform based on the MCIN to prove the effectiveness and soundness of the MCIN modeling.

We intend to continue our research in the following two directions:

(1) implement the ADI-based multi-agent architecture of the MCIN; and

(2) enhance the function of the prototype system and deploy it to industrial application.

\section{References}

Milgram, S. (1967), "The small world problem”, Psychology Today, Vol. 2, pp. 60-67.

Nakamoto, S. (2008), Bitcoin: A Peer-to-Peer Electronic Cash System, available at: bitcoin.org (accessed 4 January 2017).

Shen, J. (2015), A Study on Cyber-Anima Model and Its Application in Intelligent Recommendation: [D], Fudan Universtiy.

Robinson, L. (2007), "The cyberself: the self-ing project goes online, symbolic interaction in the digital age", New Media \& Society, Vol. 9, pp. 93-110.

Wen, J., Ming, K., Wang, F., Huang, B. and Ma, J. (2009), “Cyber-I: vision of the individual's counterpart on cyberspace”, DASC, IEEE Computer Society, pp. 295-302.

\section{Further reading}

Eskins, D. and Sanders, W. (2011), "The multiple-asymmetric-utility system model: a framework for modeling cyber-human systems", Eighth International Conference on Quantitative Evaluation of Systems, QEST, pp. 233-242.

\section{Corresponding author}

Jianping Shen can be contacted at: shenjianping@tsinghua.edu.cn

For instructions on how to order reprints of this article, please visit our website: 